\title{
COMPUTATIONAL COMPLEXITY OF CLASSICAL PROBLEMS FOR HEREDITARY CLIQUE-HELLY GRAPHS
}

Flavia Bonomo

Departamento de Computación

Facultad de Ciencias Exactas y Naturales

Universidad de Buenos Aires

Buenos Aires - Argentina

fbonomo@dc.uba.ar

\section{Guillermo Durán *}

Departamento de Ingeniería Industrial

Facultad de Ciencias Físicas y Matemáticas

Universidad de Chile

Santiago - Chile

gduran@dii.uchile.cl

* Corresponding author/autor para quem as correspondências devem ser encaminhadas

Recebido em 04/2004; aceito em 09/2004

Received April 2004; accepted September 2004

\begin{abstract}
A graph is clique-Helly when its cliques satisfy the Helly property. A graph is hereditary clique-Helly when every induced subgraph of it is clique-Helly. The decision problems associated to the stability, chromatic, clique and clique-covering numbers are NP-complete for clique-Helly graphs. In this note, we analyze the complexity of these problems for hereditary clique-Helly graphs. Some of them can be deduced easily by known results. We prove that the clique-covering problem remains NP-complete for hereditary clique-Helly graphs. Furthermore, the decision problems associated to the clique-transversal and the clique-independence numbers are analyzed too. We prove that they remain NP-complete for a smaller class: hereditary clique-Helly split graphs.
\end{abstract}

Keywords: computational complexity; hereditary clique-Helly graphs; split graphs. 


\section{Introduction}

All graphs in this paper are finite, without loops or multiple edges. For a graph $G$ we denote by $V(G)$ and $E(G)$ the vertex set and the edge set of $G$, respectively.

A graph is complete if every pair of vertices is connected by an edge. A complete in a graph $G$ is a subset of pairwise adjacent vertices of $G$. A clique in a graph is a complete maximal under inclusion. The clique number of a graph $G$ is the cardinality of a maximum clique of $G$ and is denoted by $\omega(G)$.

The chromatic number $\chi(G)$ of a graph $G$ is the smallest number of colours that can be assigned to the vertices of $G$ in such a way that no two adjacent vertices receive the same colour.

A clique cover of a graph $G$ is a subset of cliques covering all the vertices of $G$. A cliquetransversal is a set of vertices intersecting all the cliques of $G$. The clique-covering number $k(G)$ and the clique-transversal number $\tau_{C}(G)$ are the cardinalities of a minimum clique cover and a minimum clique-transversal of $G$, respectively.

A stable set in a graph $G$ is a subset of pairwise non-adjacent vertices of $G$. A cliqueindependent set is a subset of pairwise disjoint cliques of $G$. The stability number $\alpha(G)$ and the clique-independence number $\alpha_{C}(G)$ are the cardinalities of a maximum stable set and a maximum clique-independent set of $G$, respectively.

Consider a finite family of non-empty sets. The intersection graph of this family is obtained by representing each set by a vertex, two vertices being connected by an edge if and only if the corresponding sets intersect. The clique graph $K(G)$ of $G$ is the intersection graph of the cliques of $G$.

A family $S$ of subsets satisfies the Helly property when every subfamily of $S$ consisting of pairwise intersecting subsets has a common element. A graph is clique-Helly $(\mathrm{CH})$ when its cliques satisfy the Helly property. A graph $G$ is hereditary clique-Helly $(\mathrm{HCH})$ when $H$ is clique-Helly for every induced subgraph $H$ of $G$. These graphs have been characterized in [Pr93] as the graphs which contains none of the four graphs in Figure 1 as an induced subgraph. This characterization leads to a polynomial time recognition algorithm for hereditary clique-Helly graphs.

An interesting survey on clique-Helly and hereditary clique-Helly graphs appears in [Fa02].
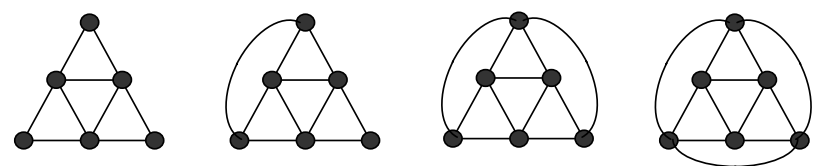

Figure 1 - Hajös graphs

A graph is split if its vertices can be partitioned into a clique and a stable set.

The neighborhood of a vertex $v$ in a graph $G$ is the set $N(v)$ consisting of all the vertices that are adjacent to $v$. The closed neighborhood of $v$ is $N[v]=N(v) \cup\{v\}$. A vertex $v$ of $G$ is called simplicial when $N[v]$ is a complete of $G$, and universal when $N[v]=V(G)$. 
It is easy to see that the decision problems associated to the stability, chromatic, clique and cliquecovering numbers are NP-complete for clique-Helly graphs. The reduction is trivial: we have to add a universal vertex to the general graph $G$ in order to generate a clique-Helly graph $G^{+}$.

However, $\omega(G)$ can be obtained in polynomial time for $\mathrm{HCH}$ graphs. The number of cliques is bounded by the number of edges [Pr93] and all the cliques can be generated in $O(n m k)$, where $m$ is the number of edges, $n$ the number of vertices and $k$ the number of cliques of the graph [TIAS77].

The stable set and the colorability problems remain NP-complete for $H C H$ graphs. These results are direct corollaries of the NP-completeness of these problems for triangle-free graphs [Pol74], [MP96]. For triangle-free graphs, a subclass of $\mathrm{HCH}$ graphs, the cliquecovering number can be obtained in polynomial time [GJ79].

So, the following question arises naturally: what happens with the complexity of the cliquecover problem for hereditary clique-Helly graphs?

The decision problems associated to the problems of finding the clique-independence number and the clique-transversal number are NP-complete [CFT93] and NP-hard [EGT92], respectively. This last problem is not known to be in NP, in fact the problem of determining if a subset of vertices is a clique-transversal is NP-hard [DLS02].

The clique-transversal problem is NP-complete for $H C H$ graphs. Again, this result is a consequence of the NP-completeness of this problem for triangle-free graphs. In this class of graphs, the clique-transversal problem is equivalent to vertex cover, and vertex cover is NP-complete for triangle-free graphs [Pol74]. Remember that in this case the problem is in $\mathrm{NP}$ for the property of $\mathrm{HCH}$ graphs above mentioned. This problem remains NP-complete for split graphs [GP00].

However, the clique-independence number can be obtained in polynomial time for trianglefree graphs, because it is equivalent in this case to maximum matching. This problem is NP-complete for split graphs [GP00] but, to our knowledge, it was not known its complexity for clique-Helly graphs.

Again, the following question appears naturally: what happens with the complexity of the clique-independence problem for hereditary clique-Helly graphs?

In this note, we prove that clique-cover and clique-independence problems remain $\mathrm{NP}$-complete for $\mathrm{HCH}$ graphs. Additionally, it is proved that clique-transversal and cliqueindependence problems remain NP-complete for a smaller class: the intersection between $\mathrm{HCH}$ and split graphs.

\section{Preliminaries}

There are some relations between the parameters defined in the introduction in a graph $G$ and its clique graph $K(G)$.

Theorem 2.1 Let $G$ be a graph. Then:

(i) $\quad \alpha_{C}(G)=\alpha(K(G))$.

(ii) If $G$ is a clique-Helly graph then $\tau_{C}(G)=k(K(G))$. 
Proof: (i) It follows from the fact that independent cliques of $G$ correspond to non adjacent vertices in $K(G)$, and conversely, non adjacent vertices in $K(G)$ correspond to independent cliques in $G$.

(ii) Let $v_{1}, \ldots, v_{\tau_{C}(G)}$ be a clique-transversal set of $G$. For each $i$, analyze the vertices in $K(G)$ corresponding to the cliques in $G$ that contain the vertex $v_{i}$. They form a complete of $K(G)$. This complete must be included in some clique $L_{i}$ of $K(G)$. Observe that these cliques $L_{i}\left(i=1, \ldots, \tau_{C}(G)\right)$ are not all necessarily different. Let us see that these at most $\tau_{C}(G)$ cliques are a clique cover of $K(G)$. Let $w$ be a vertex of $K(G)$. Then $w$ corresponds to some clique $M_{w}$ of $G$. As the set $v_{1}, \ldots, v_{\tau_{C}(G)}$ intersects all the cliques of $G$, there is some vertex $v_{j}$ that belongs to $M_{w}$. This means that the corresponding vertex of $M_{w}$ in $K(G)$ belongs to the clique $L_{j}$, i.e, $w \in L_{j}$. Then, the size of the minimum clique cover of $K(G)$ is at most the size of this clique cover which is at most $\tau_{C}(G)$.

All we need to prove is that if $G$ is clique-Helly, then $\tau_{C}(G) \leq k(K(G))$. By the Helly property, each clique $L$ of $K(G)$ has an associated vertex $v_{L}$ in $G$ such that $v_{L}$ belongs to all the cliques of $G$ corresponding to the vertices of $L$ in $K(G)$.

Let $L_{1}, \ldots, L_{k(K(G))}$ be a clique cover of $K(G)$. Let $v_{L_{1}}, \ldots, v_{L_{k(K(G))}}$ be the vertices in $G$ associated to those $k(K(G))$ cliques. Let us see that they form a clique-transversal set of $G$. Let $M$ be a clique of $G$ and $w_{M}$ its corresponding vertex in $K(G)$. Then there is an index $j$ such that $w_{M}$ belongs to the clique $L_{j}$ in $K(G)$. It follows that $v_{L_{j}}$ belongs to $M$ in $G$.

Let $M_{1}, \ldots, M_{k}$ and $v_{1}, \ldots, v_{n}$ be the cliques and vertices of a graph $G$, respectively. A clique matrix $A_{G} \in \mathbb{R}^{k \times n}$ of $G$ is a 0-1 matrix whose entry $a_{i j}$ is 1 if $v_{j} \in M_{i}$, and 0 , otherwise.

Another characterization of $\mathrm{HCH}$ graphs is the following [Pr93]: a graph $\mathrm{G}$ is $\mathrm{HCH}$ if and only if $A_{G}$ does not contain a vertex-edge incidence matrix of a triangle as a submatrix.

Let $M_{1}, \ldots, M_{k}$ and $v_{1}, \ldots, v_{n}$ be the cliques and vertices of a graph $G$, respectively. Define the graph $H(G)$ where $V(H(G))=\left\{q_{1}, \ldots, q_{k}, w_{1}, \ldots, w_{n}\right\}$, each $q_{i}$ corresponds to the clique $M_{i}$ of $G$, and each $w_{j}$ corresponds to the vertex $v_{j}$ of $G$. The edges of $H(G)$ are the following: the vertices $q_{1}, \ldots, q_{k}$ induce the graph $K(G)$, the vertices $w_{1}, \ldots, w_{n}$ are a stable set and $w_{j}$ is adjacent to $q_{i}$ if and only if $v_{j}$ belongs to the clique $M_{i}$ in $G$.

Let $A \in \mathbb{R}^{n \times m}$ and $B \in \mathbb{R}^{n \times k}$ be two matrices. We define the matrix $A \mid B \in \mathbb{R}^{n \times(m+k)}$ as $(A \mid B)(i, j)=A(i, j)$ for $i=1, \ldots, n, j=1, \ldots, m$ and $(A \mid B)(i, m+j)=B(i, j)$ for $i=1, \ldots, n$, $j=1, \ldots, k$. Let $I_{n}$ be the $n \times n$ identity matrix.

Theorem 2.2 [Ham68] Let $G$ be a clique-Helly graph and $H(G)$ as it is defined above. Then the cliques of $H(G)$ are $N\left[w_{i}\right]$ for each $i, w_{i}$ is a simplicial vertex of $H(G)$ for every $i$, and $K(H(G))=G$. 
Corollary 2.1 Let $G$ be a clique-Helly graph, $|V(G)|=n$. Then $A_{H(G)}=A_{G}^{t} \mid I_{n}$.

Proof: It follows directly from the fact that $N\left[w_{i}\right](i=1, \ldots, n)$ are the cliques of $H(G)$ and each clique contains the vertex $w_{i}$ and the vertices $q_{j}$ whose corresponding cliques $M_{j}$ contain the vertex $v_{i}$ in $G$.

This corollary leads us to prove the following result:

Theorem 2.3 Let $G$ be an $H C H$ graph. Then $H(G)$ is $H C H$.

Proof: Let $G$ be an $H C H$ graph, $|V(G)|=n$. By Corollary 2.1, $A_{H(G)}=A_{G}^{t} \mid I_{n}$. Let us suppose that $A_{H(G)}$ contains a vertex-edge incidence matrix of a triangle as a submatrix. Since it has two 1's in each column, it must be a submatrix of $A_{G}^{t}$, but then $A_{G}$ contains a vertex-edge incidence matrix of a triangle as a submatrix, which is a contradiction.

\section{Clique Cover}

The decision problem associated to the problem of finding the clique-covering number of a graph is the following:

\section{CLIQUE COVER}

INSTANCE: A graph $G=(V, E)$, a positive integer $K \leq|V|$.

QUESTION: Are there $k \leq K$ cliques of $G$ covering all the vertices of $G$ ?

To prove that CLIQUE COVER is NP-complete for $\mathrm{HCH}$ graphs, we will use that the following problem is NP-complete [GJ79]:

\section{EXACT COVER BY 3-SETS (X3C)}

INSTANCE: A set $X$ such that $|X|=3 q$ and a collection $C$ of 3-element subsets of $X$.

QUESTION: Does $C$ contain an exact cover (by $q$ sets) of $X$ ?

Theorem 3.1 The problem CLIQUE COVER is NP-complete for HCH graphs.

Proof: The transformation from X3C to CLIQUE COVER on $H C H$ graphs is based on the transformation given in [GJ79] from X3C to PARTITION INTO TRIANGLES and is the following: let the set $X$ with $|X|=3 q$ and the collection $C$ of 3-element subsets of $X$ be an arbitrary instance of X3C. We will construct an $H C H$ graph $G=(V, E)$, with $|V|=3 q$, such that $G$ has a clique cover of size at most $q^{\prime}$ if and only if $C$ contains an exact cover of $X$.

We will replace each subset $c_{i}=\left\{x_{i}, y_{i}, z_{i}\right\}$ in $C$ by the graph of Figure 2. Let $E_{i}$ be the set of 18 edges of the graph corresponding to $\left\{x_{i}, y_{i}, z_{i}\right\}$. 


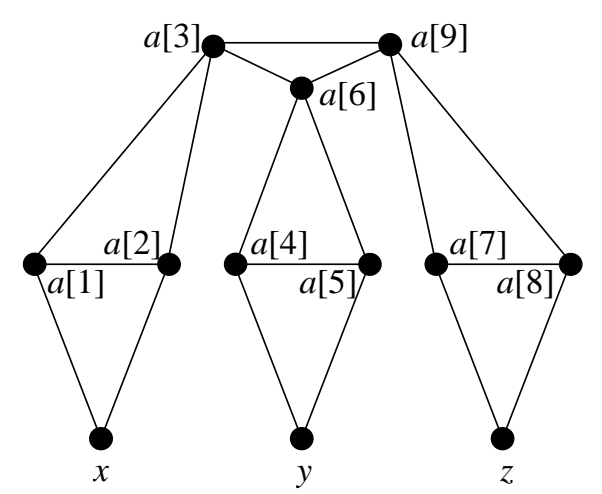

Figure 2 - Local replacement for $c=\{x, y, z\}$ in $C$ for transforming X3C to CLIQUE COVER.

Thus $G=(V, E)$ is defined by

$$
V=X \cup \bigcup_{i=1}^{|C|}\left\{a_{i}[j]: 1 \leq j \leq 9\right\}, \quad E=\bigcup_{i=1}^{|C|} E_{i}
$$

It is easy to see that $G$ does not contain any graph of Figure 1 as an induced subgraph, thus $G$ is an $H C H$ graph, $|V|=|X|+9|C|\left(q^{\prime}=q+3|C|\right)$ and the transformation can be made in polynomial time. Figure 3 shows an example of this transformation from an instance of $\mathrm{X} 3 \mathrm{C}$ to an instance of CLIQUE COVER.

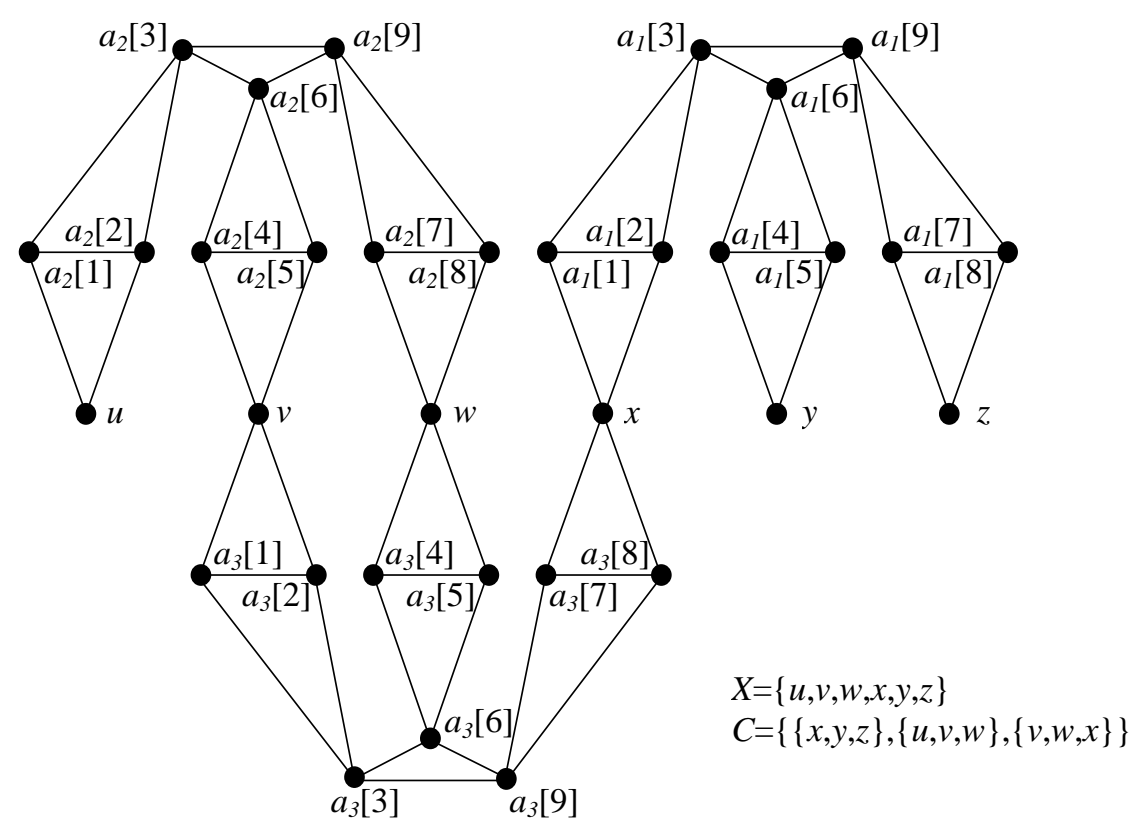

Figure 3 - Transformation from an instance of X3C to an instance of CLIQUE COVER. 
Let us suppose that $C$ contains an exact cover of $X$, then we construct a clique cover of $G$ of size $q$, by taking for each $1 \leq i \leq|C|$

$$
\left\{a_{i}[1], a_{i}[2], x_{i}\right\},\left\{a_{i}[4], a_{i}[5], y_{i}\right\},\left\{a_{i}[7], a_{i}[8], z_{i}\right\},\left\{a_{i}[3], a_{i}[6], a_{i}[9]\right\},
$$

whenever $c_{i}=\left\{x_{i}, y_{i}, z_{i}\right\}$ is in the exact cover and

$$
\left\{a_{i}[1], a_{i}[2], a_{i}[3]\right\},\left\{a_{i}[4], a_{i}[5], a_{i}[6]\right\},\left\{a_{i}[7], a_{i}[8], a_{i}[9]\right\},
$$

otherwise.

Let us now suppose that $G$ has a clique cover of size at most $q^{\prime}$. Since the cliques of $G$ are triangles, the number of cliques in the clique cover must be $q$ ' and each vertex of $G$ must be covered exactly once.

In the graph of Figure 2, the only two ways of covering by triangles each vertex $a_{i}[j]$ $(j=1, \ldots, 9)$ exactly once are the above mentioned, covering or not $x_{i}, y_{i}$ and $z_{i}$, respectively. Then the exact cover of $X$ is given by choosing those $c_{i} \in C$ such that $\left\{a_{i}[3], a_{i}[6], a_{i}[9]\right\}$ belongs to the clique cover of $G$.

Finally, the membership in NP for the restricted problem follows from that for the general problem.

\section{Clique Transversal and Clique-Independent Set}

The decision problems associated to the problems of finding the clique-independence number and the clique-transversal number of a graph, respectively, are the following:

\section{CLIQUE-INDEPENDENT SET}

INSTANCE: A graph $G=(V, E)$, a positive integer $K \leq|V|$.

QUESTION: Is there a set of $K$ or more pairwise disjoint cliques of $G$ ?

\section{CLIQUE-TRANSVERSAL}

INSTANCE: $G=(V, E)$, a positive integer $K \leq|V|$.

QUESTION: Is there a set of $K$ or fewer vertices of $G$ intersecting all the cliques of $G$ ?

Theorem 4.1 The problems CLIQUE-TRANSVERSAL and CLIQUE-INDEPENDENT SET are NP-complete for $\mathrm{HCH}$ split graphs.

Proof: We will show a polynomial time transformation from CLIQUE COVER on $\mathrm{HCH}$ graphs (by Theorem 3.1 it is NP-complete) to CLIQUE-TRANSVERSAL on $H C H$ split graphs.

Define the graph $G^{+}$where $V\left(G^{+}\right)=V(G) \cup\{u\}, V(G)$ induces the graph $G$ and $u$ is a universal vertex. Since for any graph $G$ all the cliques of $G^{+}$share the vertex $u$, the graph $K\left(G^{+}\right)$is complete and thus the graph $H\left(G^{+}\right)$is a split graph. 
Let $\mathrm{G}$ be an $\mathrm{HCH}$ graph. As the set of cliques of an $\mathrm{HCH}$ graph has polynomial size and can be computed in polynomial time, $H\left(G^{+}\right)$can be built in polynomial time. By Theorem 2.3, since $G^{+}$is an $H C H$ graph, $H\left(G^{+}\right)$is an $H C H$ graph. By Theorem $2.2 K\left(H\left(G^{+}\right)\right)=G^{+}$, and by Theorem $2.1 k(G)=k\left(G^{+}\right)=\tau_{C}\left(H\left(G^{+}\right)\right)$. Finally, the problem of determining if a subset of vertices is a clique-transversal is solvable in polynomial time for $\mathrm{HCH}$ graphs, and therefore CLIQUE-TRANSVERSAL is NP-complete for $\mathrm{HCH}$ split graphs.

In a similar way, using the equality $\alpha(G)=\alpha\left(G^{+}\right)=\alpha_{C}\left(H\left(G^{+}\right)\right)$instead of $k(G)=$ $k\left(G^{+}\right)=\tau_{C}\left(H\left(G^{+}\right)\right)$, and the NP-completeness of the STABLE SET problem for $H C H$ graphs, CLIQUE-INDEPENDENT SET is NP-complete for $\mathrm{HCH}$ split graphs.

Corollary 4.1 The problem CLIQUE-INDEPENDENT SET is NP-complete for HCH graphs.

\section{Acknowledgements}

To the anonymous referees for their careful reading and valuable suggestions which improved this work.

The first author was partially supported by UBACyT Grant X184, PICT ANPCyT Grant 11-09112 and PID CONICET Grant 644/98, Argentina and "International Scientific Cooperation Program CONICyT/SETCIP”, Chile-Argentina.

The second author was partially supported by FONDECyT Grant 1030498 and Millennium Science Nucleus "Complex Engineering Systems", Chile and "International Scientific Cooperation Program CONICyT/SETCIP”, Chile-Argentina.

\section{References}

[CFT93] Chang, M.; Farber, M. \& Tuza, Z. (1993). Algorithmic aspects of neighbourhood numbers. SIAM Journal on Discrete Mathematics, 6, 24-29.

[DLS02] Durán, G.; Lin, M. \& Szwarcfiter, J. (2002). On clique-transversal and cliqueindependent sets. Annals of Operations Research, 116, 71-77.

[EGT92] Erdös, P.; Gallai, T. \& Tuza, Z. (1992). Covering the cliques of a graph with vertices. Discrete Mathematics, 108, 279-289.

[Fa02] Farrugia, A. (2002). Clique-Helly graphs and hereditary clique-Helly graphs, a minisurvey. Manuscript.

[GJ79] Garey, M. \& Johnson, D. (1979). Computers and Intractability: A Guide to the Theory of NP-Completeness. Freeman and Company, San Francisco.

[GP00] Guruswami, V. \& Pandu Rangan, C. (2000). Algorithmic aspects of cliquetransversal and clique-independent sets. Discrete Applied Mathematics, 100, 183-202.

[Ham68] Hamelink, R. (1968). A partial characterization of clique graphs. Journal of Combinatorial Theory, Series B, 5, 192-197. 
[MP96] Maffray, F. \& Preissmann, M. (1996). On the NP-completeness of the $k$-colorability problem for triangle-free graphs. Discrete Mathematics, 162, 313-317.

[Pol74] Poljak, S. (1974). A note on stable sets and colorings of graphs. Commentationes Mathematicae Universitatis Carolinae, 15, 307-309.

[Pr93] Prisner, E. (1993). Hereditary clique-Helly graphs. Journal of Combinatorial Mathematics and Combinatorial Computing, 14, 216-220.

[TIAS77] Tsukiyama, S.; Idle, M.; Ariyoshi, H. \& Shirakawa, Y. (1977). A new algorithm for generating all the maximal independent sets. SIAM Journal on Computing, 6(3), 505-517. 